\title{
Topologie der Heimat zwischen Imaginärem, Mythos und begrifflicher Sprache Paratopos, Utopie-Dystopie-Heterotopie und Transtopisches
}

\author{
In Erinnerung an Gerda E. Moser
}

\author{
Alice Pechriggl \\ Universität Klagenfurt (Austria)
}

\begin{abstract}
The article examines Heimat as a multilayered signification and affectively invested phenomenon in the context of the related social imaginary. A topologic perspective elucidating the role of topos in the Heimat imaginary is central to this examination deployed around the idea that Heimat is a paratopos, a place nearby, protecting against the Other/s. Although "Heimat" is a German term which cannot be translated easily, something of the phenomenon seems to exist in cultures other than the Germanophone. It is part of philosophical culture, as will be shown with the help of relevant philosophers. Political imaginaries are also often founded by the "Heimat" phenomenon by way of an origin myth narrating the beginnings of a society or community as homeland or home country, patris, sometimes metropolis, patrie,
\end{abstract}

Heimat etc. After a brief look back into the Ancient Greek imaginary of the patris, the article discusses the gender asymmetry in the topology of "Heimat" and what the author calls the screen imaginary of femininity. This section is followed by a group-psychoanalytic consideration of affective ambivalence and the defense mechanism of splitting at work in the collective imaginary of Heimat and nationalism. Last but not least, the article tries to sketch some "transtopic" ways out of these xenophobic and martial aspects of Heimat as constructed "against" the foreigner as the enemy.

Keywords: Paratopos, self-othering, screen imaginary offemininity, splitting

(c) Alice Pechriggl; alice.pechriggl@aau.at

Colloquium: New Philologies, Volume 6, Issue 1 (2021)

doi: 10.23963/cnp.2021.6.1.9

Stable URL: https://colloquium.aau.at/index.php/Colloquium/article/view/153

This work is licensed under a Creative Commons Attribution 4.0 International License (CC BY 4.0). 


\section{Einleitung}

Heimat ist kein philosophischer Begriff, doch es wird seit langem philosophisch-begrifflich über dieses Wort und das Phänomen, das es bezeichnen soll, nachgedacht. Wenn in diesem Sinn Heimat als ein Ort, der keiner ist, beschrieben werden kann - als eine Zeit, die nie war und auch nicht kommt, dann deshalb, weil die meisten im deutschsprachigen Raum heimischen Menschen etwas damit verbinden; und zwar etwas, das zwischen Existenz und Inexistenz, zwischen Fiktion und Realem angesiedelt ist. Es ist dies eine Wirklichkeit, die Cornelius Castoriadis eine zentrale gesellschaftlich-imaginäre Bedeutung nennen würde. Für den Erfinder des Begriffs des "gesellschaftlichen Imaginären“ verdichten sich in solchen zentralen Bedeutungen Vorstellungen, Wünsche und Affekte zu einem instituierten, vor allem aber zu einem effektiv erlebten Phänomen, das die einfache Gegenüberstellung von fiktiv Imaginärem und real Gegenständlichem durchkreuzt. Ohne Betrachtung der Psyche und ihrer auf kollektiver Ebene relevanten Dimensionen ist es nunmehr schwer, diesem Phänomen gerecht zu werden. Deshalb ist meine Zugangsweise, wie immer in solchen Fällen, eine philosophisch-gruppen/psychoanalytische und eine chiasmatische (von $\chi$ - die Gegensatzpaare über Kreuz legende).

Dass das Imaginäre wirklich ist, gilt in ganz besonderer Weise für den Begriff „Heimat" und das Phänomen, das er zu bezeichnen aufgerufen wird. Damit kann Heimat philosophisch als ein konkret transzendentales Phänomen für die einzelnen Menschen gefasst werden, ähnlich wie "Schicksal“ oder „Muttersprache“, auch wenn Heimat ein deutsches Wort ist und seine Bedeutung keineswegs in allen Kulturen existiert. Doch bleiben wir bei Deutsch: Die Anrufung der „Heimat“, und die meisten germanophonen Menschen fühlen sich zu einer solchen zugehörig, evoziert Affekte, Wünsche aber auch Angst und Abneigung. Es geht dabei um die Angst des „Heimatverlustes“ und um Wünsche als Sehnsucht nach Rückkehr in Form von „Heimweh“, aber auch um heftige Ablehnung, insbesondere, wenn Heimat zum nationalistischen oder gar faschistischen Topos der Kriegspropaganda oder zum politischen Kampfbegriff gegen Asylsuchende, Migrant_innen oder andere nicht Autochthone gemacht wird. Es kann aber auch ganz persönliche Gründe geben, warum jemand gegen (seine) „Heimat“ eine Abneigung hegt oder diesen Begriff für sich ablehnt. Wo ist die Philosophie, wenn es um Heimat geht? Die Philosoph_innen? Ich werde dazu anhand einer kleinen Auswahl einen Streifzug versuchen.

Mit Blick auf die Abwesenheit von „Heimat“ in anderen Sprachen, werde ich auch der Bedeutung der patris oder patria in der Genese von „Heimat“, auf die Geschlechterasymmetrie im gesellschaftlichen Imaginären und in der Praxis der Einsetzung dieser - nicht nur im deutschsprachigen Raum - so zentralen Bedeutung eingehen, bevor ich 
mich zum Schluss wieder der germanophonen und als solcher unübersetzbaren Verfasstheit von Heimat und ihrem Überschreitungspotential zuwende, das ich „transtopisch“ nenne.

\section{Heimat als „Paratopos“ am Beispiel Österreichs und darüber hinaus}

Aus philosophisch-kulturwissenschaftlicher Perspektive ist Heimat jedoch nicht nur kollektiv verbindend und identifikatorisch relevant, sie ist vor allem ein Topos (gr. für Ort, Platz, aber auch weibliches Geschlechtsorgan und im übertragenen Sinn Allgemeinpatz, lieu commun). Ich würde sie zuerst einen Paratopos ${ }^{1}$ nennen, der immer schon im Zeichen der Nachträglichkeit steht, wie alle psychoanalytisch interessanten Phänomene. Ich meine damit einen Topos, der kein realer Ort ist, d.h. kein Ort auf irgendeiner geographischen Karte, an dem gestanden oder an den gegangen werden könnte, oder zumindest nicht nur, denn etwas von der Örtlichkeit und ihrer Verortung bleibt an der Heimat immer haften. Im „Paratopos“ steckt das Gegenstück und der Widersinn, aber auch die Beiläufigkeit des griechischen Suffix para-: Er ist es (Topos im eigentlichen Sinn) und er ist es nicht, ein bei/nahe-Ort also und kein Nicht-Ort wie der A-Topos, das Ungewöhnliche oder die U-topie, die mit dem Griechischen Topos ohnehin nur noch wenig zu tun hat.

Paratopos also, oder „Paraort“ (analog zu Abort). Er verweist auf einen Widersinn der Örtlichkeit, denn die Heimat ist zwar meist territorial verortbar, aber zugleich weit davon entfernt, sich in dieser Verortung zu erschöpfen, ja sie geht in extremis sogar soweit, ohne Boden, ohne chôra, (gr. für Territorium, Land) Bestand zu haben: Dafür steht Athen während der Schlacht von Salamis gegen die Perser ebenso Pate wie das jüdische Volk in der Diaspora. ${ }^{2}$

1 Paratop, engl. paratope, gr. paratopos, bezeichnet in der Genetik jene Stelle eines Antikörpers, an der dieser an ein Antigen bindet, genauer an dessen Epitop. Auch wenn wir in einer Pandemie leben und die Immunologie bzw. Epidemiologie schon seit der Renaissance für die Staatsraison, insbesondere im Zeichen der organologischen Metapher vom Volkskörper, strukturierend war, möchte ich hier keine solchen Verbindungen ziehen.

2 „Die Polis ist ihre Männer, polis gar andres“ und alle, die auf diesen Befehl des Themistokles hin auf die verfügbaren Schiffe evakuiert werden sollen, kurz bevor die Xerxes-Armee die Stadt in Schutt und Asche legt (siehe Thuk., Pelop. Krieg, Jahr 413). Was die Diaspora angeht, so prägt der Auszug aus Ägypten nicht nur die jüdische Religions- und Kulturgemeinschaft, sondern auch die auf ihr aufbauenden abrahamitischen, allerdings nicht in vergleichbar tragischer Weise. Andere, vor allem moderne Phänomene der Migration, die oftmals in einem inflationären Gebrauch des Wortes als Diaspora bezeichnet werden, sind hier nicht automatisch mitgemeint. Besonders entbehrlich und von sozialwissenschaftlich zweifelhaftem Wert sind Listen, zumal auf ihnen stets bestimmte aus ihrer „Heimat“ vertriebene Gruppen nicht aufscheinen (es wird immer irgendeine Gruppe vergessen worden sein ...) Dennoch sind die globalen Vertreibungsphä- 
Dieser Paraort „Heimat“ trägt immer auch schon jenes Gegenstück in sich, über das es sich mit der Nichtheimat, der Fremde verbindet. Das liegt zum einen an der Dialektik des Phänomens, die darin besteht, dass Heimat ohne Fremde überhaupt nicht zur Wirkung kommen könnte, ja vielleicht nicht einmal zu irgendeiner Bedeutung gelangt wäre.

Zum Paraort gehört eine Parazeit, denn die Heimat ist in ihrer mythischen Verfasstheit irgendwie zeitlos und dennoch an den Mythos ihrer zu einem bestimmten Zeitpunkt beginnenden Auto/biographie gebunden. Es ist dies der die Existenz eines Kollektivs begründende Herkunftsmythos, der als immer auch fiktionale Erzählung den weiteren Verlauf der Geschichte dieser oder jener Heimat im kollektiven Imaginären fundiert.

Zumeist steht ein Gründungsereignis an dieser mythischen Stelle, eine Rettung, eine Revolution, ein Friedens- oder ein Staatsvertrag. Letzteres etwa in Hugo Portischs „Zweite Republik“, die wir anlässlich von dessen Tod gerade wieder im Österreichischen Rundfunk zu sehen bekommen und die - ähnlich wie die Geschichtserzählungen der „République Française“ durch Jules Michelet, Pierre Nora und andere seit 1789 - für das Volk und seine Identifikation mit dem Staat als Heimat, als Land und als Regime verfasst und breitenwirksam ausgestrahlt wird. ${ }^{3}$ Da gerade in einem minimal demokratisch verfassten Rechtsstaat diese Art der historiographischen Erzählung einer gewissen Objektivität verpflichtet ist, wird die Geschichte mit einigen Widersprüchen und nicht nur mit ideologischen, im Sinne von Marx die Herrschenden und ihre ausbeuterische Herrschaft legitimierenden, Erbaulichkeiten versehen. Der „Opfermythos“ ist hierin zentral und der durch ihn versäumte Umgang mit dem Anschluss an Deutschland, an Nazideutschland, an Deutschland tout court.

Der Topos des Deutschen, Großdeutschen, Deutschnationalen in Österreich, zu Beginn der Ersten Republik auch „Deutschösterreich“ genannt, ist eine Kernbedeutung des Paraorts Heimat Österreich, bis heute. Das Deutschwerden Österreichs geschah zum einen durch die Niederlage im 1. Weltkrieg und danach auf Betreiben der in der Monarchie unterdrückten slawischsprachigen Bevölkerungen; nach dem Zerfall des Reichs geschah und geschieht es zum anderen auf Kosten der in Österreich verbliebenen „Minderheiten" (Slowen_innen und Kroat_innen werden im Staatsvertrag als solche anerkannt), auf Kosten der Vielsprachigkeit, der Vielfalt und der Aufklärung darüber, was Österreich an seinen Nebenschauplätzen, in seinen düstersten Kellern, Heimaterln, Nebenlagern und

nomene zu allen Zeiten für die Frage nach der Bodenlosigkeit des Paratopos Heimat relevant. (Safran, 1991).

3 Der studierte Historiker und Journalist Portisch, den meine und spätere Generationen von Österreicher_innen als Geschichtsonkel der Nation erlebten, verstarb am 1. April 2021. Michelet gilt in Frankreich bis heute als der Historiker der Revolution, auch wenn sich rund um die 200-Jahr-Feier François Furet und Mona Ozouf an seine Stelle zu setzen suchten. Für sein Monumentalwerk wählte der selbsternannte Auto/biograph der Nation Nora den Titel „Lieux de mémoires“ (Erinnerungsorte, wobei mémoire eher das Gedächtnis und commémoration die Erinnerung bezeichnet). 
Politsümpfen sonst noch alles ist. Auch wenn die Österreicher_innen das mehrheitlich lieber nicht wissen wollen, gilt es in einem Rechtsstaat ein differenziertes Geschichtsverständnis zu vermitteln und ein wenig von der sozialpsychologischen Gewissheit umzusetzen, dass ein Umgang mit diesen düsteren Seiten der Geschichte sich geziemt und für ruhigeren Schlaf in Gegenwart und Zukunft sorgt. Auch da hilft der Paratopos Heimat, der die Ambivalenz aufrecht zu erhalten vermag und zugleich eine abwägende, kritisch sortierende Einschätzung wenn auch nicht verunmöglicht, so doch ganz gut behindert.

Er erlaubt es der Bevölkerung, die im Tourismus schon früh, in den Universitäten erst in jüngerer Zeit vorherrschenden Unterwerfungsgesten gegenüber den oftmals schwadronierend überheblichen, weil reineren und besseren Deutschen unablässig einzuüben; Gesten hin zu diesen zahlenden, und immer öfter auch gut bezahlten „Gästen“, die ja selbst keinen Minderheiten in ihrer heimatlichen Mitte Fremdsprachenrechte einräumen zu müssen meinen (mit dem kolonialistischen Erbe geht es jetzt erst langsam los). Doch statt eine etwas gründlichere Schau in die großdeutsch/österreichischen Keller deutscher Heimattreue vor sowie während der Zweiten Republik zu wagen, wird das gar nicht harmlose Ressentiment gegen die „Piefke“ kultiviert, diese eigentlichen Täter (wäre da nicht der oberste Täter Adolf Hitler und seine zahlenmäßig überrepräsentierten Landsmänner) - ein Ressentiment, das zur österreichischen Heimat gehört wie Hans Krankls Tor zum 3:2 in Cordoba. ${ }^{4}$

Der Paratopos wirkt auch dort, wo der Fremdenhass zum entsublimierten Bruderhass wird und wo dessen Ausagieren und die dazugehörigen Ersatzhandlungen die Wut und Trauer um die verlorenen Sprachen und Menschen immer noch erfolgreich an die Kellerwand spielen. ${ }^{5}$ Dieser paratopische Zug des verinnerlichten und doch abgespaltenen Deutschnationalen im Herzen des zerfallenen aber multilingual nachhinkenden Vielvölkerstaats Österreich ist nur einer der vielen Heimathüte, aber als die Spitze eines grenzüberschreitenden Schneebergs tiefer Ambivalenz, welche „Heimat“ als solche prägt, ist er zentral. Diese Ambivalenz manifestiert sich in der Struktur des Heimatimaginären, aber sie vermag auch unvorhersehbare Neuformationen in ihm hervorzubringen. Der Freudsche Begriff der Abwehr kann beim Verständnis dieser Phänomene als ein Werkzeug fungieren, das ich mit einer philosophischen Chronotopologie der Heimat zu ver-

4 Fußballmatch Österreich gegen Deutschland bei der WM 1978, in dem Deutschland wider Erwarten unterlag. Die Feindseligkeit geht nicht nur auf den Opfermythos oder auf das als arrogant wahrgenommene Benehmen deutscher Schitourist_innen in den 1970er-Jahren zurück, sondern auch auf die Kriege zwischen Österreich und Preußen, die Feindseligkeit zwischen Friedrich II. und Maria Theresia etc.; das österreichische Heimatimaginäre transportiert sie also schon länger, sie wird aber meist nicht bewusst als solche wiederbelebt.

5 Es gilt nicht nur von Wien, die auch als Hauptstadt des Balkans bezeichnet wird, dass fast jede_r dort zumindest einen slawisch- oder ungarischsprachigen Vorfahren hat. Es stimmt wohl auch für Teile Kärntens, des Burgenlandes und der Steiermark. 
knüpfen suche, denn wir haben es hier nicht nur mit Mythos und Imaginärem, sondern mit Mystifizierungen zu tun, die nicht durch aussagenlogische, geschweige denn durch formallogische Analysis allein erhellt werden können.

„Heimat“ als Topos mit der Herkunfts- und Ankunftsfrage zu verknüpfen zieht unverzüglich auch die Frage nach ihrer politischen Wirklich- und Wirksamkeit nach sich. Eine philosophisch- und historisch-anthropologische Perspektive ist im Hinblick auf die Analyse der Affektökonomie gruppen/psychoanalytisch zu wenden, denn im Mittelpunkt stehen dabei die Begriffe des gesellschaftlichen Imaginären und der „-topie“ an der Schnittstelle von Dystopie, Heterotopie und Utopie, was auf die unvermeidliche Frage nach der affektiven Ambivalenz der Heimat als eine imaginäre res extensa verweist, also auf einen imaginären Raum über den eingeengten Topos sensu strictu hinaus.

Als Produkt kollektiver Phantasie ist dieser Raum zugleich in ständiger Veränderung begriffen und wäre damit res extendenda, ein „Ding“, das dazu tendiert, sich auszubreiten, und zwar nicht selten auf Kosten anderer Räume, wie der NS-Begriff des („natürlich“ heimatlichen) „Lebensraums“ am grausamsten verdeutlichte. Überhaupt kippt im konkretistischen Imaginären der Nazis die Heimat am totalsten in die genozidäre Realisierung einer Dystopie. Dagegen waren die humanistisch-kosmopolitischen Heimatideale, für die so manche_r Intellektuelle sein Leben hingab (wie für das revolutionäre Frankreich 1789-1792, die Commune de Paris 1871, für „Griechenland“ 1821 bzw. die „Spanische Republik“ 1936) eher intellektuell sublimierte Utopien, auch wenn sie als solche nicht verwirklicht wurden. Sie glänzen allerdings durch eine eigentümlich europäische Art des Self-Otherings in der Projektion des geistigen und politischen Heimatwunschkonzerts auf eine zu rettende Republik (ich komme weiter unten auf diesen Begriff des Self-Othering zurück).

\section{Philosoph_innen in/aus der Heimat zwischen Mythos und Topos: einige Auserwählte}

Die für das Imaginäre der Heimat konstitutiven Bedeutungen ranken sich um die Polarität des Unheimlichen, des Fremden und Vertrautesten und der heimatbeschwörenden Angstabwehr. Die tiefe Angst vor dem Ausgestoßen-Sein bewohnt zwar jeden Menschen, doch nicht alle haben dagegen und darum herum eine Heimat aufgebaut. Mit dem Heimat-Imaginären bearbeiten die deutschsprachigen Menschen unablässig die affektiven Dispositionen, die mit dieser Polarität einhergehen und zugleich bevölkern sie damit ihre Kultur, auch in der Philosophie, ob die sich nun deutsch-kritisch wie bei Ernst Bloch oder Walter Benjamin, deutsch-fundamentalhinterwäldlerisch wie bei Martin Heidegger, wienerisch-sprachfetischistisch wie bei Ludwig Wittgenstein (Philoso- 
phie aus Wien) und Sigmund Freud (Metapsychologie in und aus Wien) oder kosmopolitisch wie bei Hannah Arendt (aus Deutschland) gibt, um nur die für mich relevantesten in dieser Frage zu nennen.

Während germanophone Dichtung oftmals durch die Zersetzung der Heimat diese - sublimiert - wo/anders (im Reich der literarischen Sprache) wiedererrichtet, versucht die Politik, die Heimat rhetorisch im Phantasma der Nation aufzubauen. Dagegen suchen Philosoph_innen die Funktion der Heimat in der Sprache als einem Labyrinth aus Begriffsgefügen aufzubauen, in denen vielleicht nur jene die Orientierung nicht verlieren, die am Boden der allgemeinen Wirklichkeit immer schon verloren zu sein glauben oder gar wissen (eingedenk ihrer Sterblichkeit). Das ist das spezifisch paratopische Potenzial der Philosophie, einer Welt der Begriffe, die all jenen Heimat gibt, die nicht nur im Wort, sondern auch im systematisch subversiv argumentierenden Sprachspiel zu Hause sind. Und zwar nicht erst seit Wittgenstein oder Derrida, sondern seit Heraklit und Sokrates, Platon und Aristoteles ... Und das sind die sozialen Banden dieser Heimat: Denkverwandtschaftslinien, Schulen, allesamt patrilinear wie die taoistischen Lineages, weshalb Frauen sich darin weniger beheimatet fühlen, ja immer noch eine Art „Ironie des Gemeinwesens" darstellen, um Hegels treffende Formulierung zu bemühen. (Vielleicht liegt aber gerade hierin das kritische Vermächtnis der philosophierenden Frauen, und zwar im Sinne der Philosophie und weniger in jenem des Schulenstreits zur väterlichen Ehrenrettung, wie sie seit Sokrates-Platon immer wieder das Feld beherrscht.)

Eine affektiv derart aufgeladene Bedeutung wie Heimat ist zuerst kein Begriff (Logos oder Genos) sondern ein Mythos, eine Geschichte oder Erzählung, mehr oder weniger erfinderisch gestaltet und ausgeschmückt. Bevor sie philosophisch und/oder durch politische Rhetorik zum Begriff gemacht, also gereinigt, systematisiert und aufpoliert wird, schillert sie in allen Farben folkloristischer Stimmung mit dem obligatorischen Gemeinschaftspathos, den Musik und Gesang so eindringlich verbindend vermitteln. Aus diesen schillernden Vermächtnissen schöpft die begriffliche Arbeit der Philosoph_innen, die sich der Heimat zu bemächtigen versuchen: indem sie sie kritisch beleuchten, destituieren, dekonstruieren, unterminieren, um sie im Himmel der Begriffe immer von Neuem wiederaufzubauen gemäß dem Prinzip des logon didonai (Gründe und Rechenschaft geben, möglichst stringent argumentieren, erhellen, analysieren, begreifen, etc.). Denn auch die Techniken sprachlicher Polemik und eine aus den Gerichtshöfen und den politischen Vollversammlungen in Athen stammende sublimierte Kriegführung gegen das im Volksmund affektiv aufgeladene „Daham“ (daheim) kruder Demagogen, Imperialisten und Genozidäre zielen auf einen Topos ab: den Ort der Sehnsucht (und nicht der Angst), der unerfüllten und der vormals erfüllt geglaubten. 
Ob in Blochs „Noch Nicht“ bzw. in seiner utopischen Rhapsodie Prinzip Hoffnung (Bloch 1984) oder in Benjamins genialen Hauptstadt-Spaziergängen durch die Pariser Passagen (Benjamin, 1982), die kritische Theorie deutscher Nation und jüdischen Backgrounds verweist auf die bereits geschlagenen und tödlich noch bevorstehenden Wunden antisemitischer Ausrottungspolitik im Namen einer reinen, deutsch-arischen Heimat und weist auch schon darüber hinaus. Arendt, die Jüdin im philosophischen Männerclub, oder vielmehr außerhalb desselben, welche den Topos im Aristotelischen Erbe der Theorie-Praxis-Unterscheidung zu verankern sucht, kommt gerade noch aus ihrer zerbrechenden „Heimat“ auf und davon, von ihrem Lehrer und Verführer, dem Nazi Heidegger, aber kaum los. Sie baute mit ihrem Denken weit mehr als Celansche Gräber in den Himmel der Begriffsgefüge: Pariah, Totalitarismus, Natalität, Neues und die Revolution denken „wir" seither in ihrer Nachfolge. Auch Sigmund Freud, der metapsychologisch in den geistigen Untergrund geht, bevor er sich doch noch aus seinem "lieb gewordenen“ Gefängnis Wien befreien und vor den dort bereits einmarschierten Nazis retten lässt, hinterlässt uns eine Begriffswelt, deren Erschließung und Weiterführung erst begonnen hat, obwohl sie noch zu seinen Lebzeiten in der ganzen Welt Anklang gefunden hatte.

Rettung hat in diesem semantischen Feld mit verlorener Heimat und mit Zerstörung (oder mit Katastrophe, wie es die modernen Griechen seit 1918 nennen) zu tun, nicht erst seit Friedrich Hölderlin. Freud baut bis zum letzten Atemzug für ganze Generationen von Psychotherapeut_innen, Philosoph_innen, Kulturwissenschafter_innen und sonstige Laien aus aller Welt nicht nur den Begriff des Unbewussten zu einer schier unermesslichen Un/heimat aus; er weist uns auch noch den Weg, über den wir in ihm, also im Unbewussten - erst mit Hilfe eines therapeutischen Virgil, dann selbst - uns auch heimisch machen können oder zumindest ein wenig vertrauter mit der Fremde in unserer eigenen Mitte. Freie Assoziation heißt die hierzulande längst vor Freud übliche Technik der schlüpfrig-kathartischen Wortspiele und Witze, die nach dem Ende des Naziregimes zugleich mit dem Jazz und dem freien Tanz, der freien Körperkultur und der sexuellen Revolution auch in Wien wieder Einzug hält, sich geradezu paratopisch verankert - gegen die „Ehemaligen“ (Nazis), Antisemiten, Frauenhasser und Homophobe, Klerikalfaschisten, Deutschnationale und Neonazis, die sich jetzt - im euphemistischen Neusprech der frühen Zweiten Republik - „Unabhängige“ nennen. Entgegengesetzte politische Heimaten in einer Stadt, die alles in sich aufnimmt und nicht selten wieder unter sich begräbt.

Und dann sind da noch die beiden Philosophen Ludwig Wittgenstein und Michel Foucault, die ich auch noch kurz vorstellen möchte, weil sie einander in mehr als einer Un/Heimat treffen: Wittgenstein, der Wiener, vermeintliche Gründer der zeitgenössischen amerikanischen und nunmehr Weltsystem-Philosophie, geißelt nicht nur seine Volksschüler, sondern vor allem sich selbst für seine homosexuellen Begierden, und 
verlässt die Heimat Österreich noch rechtzeitig gen Norden. Die Sprache, die in seinen Abhandlungen als aphoristische Paragraphenprosa daherkommt, und nicht nur die philosophische Sprache, wird danach nicht mehr dieselbe sein; die Gewissheit auch nicht und schon gar nicht die Illusion der Klarheit: Mimikri und Analysis der Sprache als unerreichbare Heimat. Doch der Philosoph bleibt „noch ein Gescheiter/ter“. Dagegen tritt Foucault schon selbstbewusster auf, allerdings nur implizit, was die Rechte derer angeht, die wie er anders als „straight" lieben und leben. Heute nennen sie sich im Allgemeinen queer und meinen, in Foucault eine neue Heimat gefunden zu haben. Jedenfalls verdanken wir ihm die Bereicherung der philosophisch-anthropologischen Topologie um den Begriff der Heterotopie (Foucault, 2001). Diese lädt zu einem kleinen Exkurs ein. Sie ist ein Zufluchtsort an der Schnittstelle von Imaginärem und Realem, zwischen mise en scène des Glücks und seiner kurzen mise en acte im Urlaub oder im heimlichen Kinderspiel zu Hause, und erinnert teilweise an Blochs konkrete Utopien (Baumhaus u.a.). Foucault geselle ich den aus der Heimat zweimal vertriebenen hinzu, den ich bereits erwähnte: Castoriadis wird kurz nach seiner Geburt 1922 mit seinen Eltern aus Konstantinopel von den Türken nach Athen vertrieben, von dort durch die politischen Gegner nach Paris. Der Revolutionär und Radikaldemokrat par excellence hinterließ uns nicht nur eine zeitgemäße Ontologie und Theorie des gesellschaftlichen Imaginären (Castoriadis, 1984), sondern auch das Labyrinth (Castoriadis, 1981), aus dem dereinst der kapitalistische Minotaurus verschwunden sein wird, der das Projekt der Autonomie vereitelt und unsere planetarische Lebensgrundlage zerstört. Castoriadis selbst meinte, dass es keinen Minotaurus gäbe im Labyrinth des Dädalus. Ich sehe das anders: Es gibt zahlreiche, und ein Faden wird nicht reichen, um nach deren Überwältigung wieder ins Freie zu kommen. Im Moment wissen wir nicht einmal, ob wir es mit einem Labyrinth oder nicht vielleicht doch mit einem planetaren Gefängnis zu tun haben, wo die Erde doch unser aller „Heimat“ ist: Treibhauseffekt, Pandemie, Artensterben, Atomwaffen und immer mehr Atommüll in lapidaren Umhüllungen, wenn nicht gleich ins offene Meer ...

Mit Jacques Derrida, pied noir juif ${ }^{6}$, teilt Castoriadis lange Zeit nicht nur die Betreuung der Doktoratsstudien für „Philosophie und Gesellschaftswissenschaften“ an der Ecole des Hautes Etudes en Sciences Sociales (EHESS) in Paris, sondern auch das Interesse an der Alterität. Die Destituierung des ererbten Denkens und Handelns, die er anstrebt, ist in manchem analog, obschon politisch expliziter als die begriffliche Dekonstruktion Derridas. Kombiniert vermögen beide die erbaulichen Heimatphantasmen wie kaum etwas

\footnotetext{
6 Derrida ist als Kind jüdischer Franzosen in der damaligen Kolonie Algerien geboren. Die Franzosen aus der Metropole hießen zuerst colons (Kolonisten), später pieds noirs, wörtlich „Schwarzfüße“, vor allem als sie im Zuge des Algerienkrieges nach Frankreich (France métropolitaine) zogen, wo sie noch fremder waren als in Algerien.
} 
auseinanderzulegen, vorausgesetzt ihre Bezüge zu Marx und Freud, weitere Heimatlose und doch wieder Beheimatete, werden mitgedacht.

\section{Antike Griechische Vorläufer: Chôra, Gê, Patris, Metropolis ...}

Doch kommen wir endlich zu den „Anfängen“, d.h. zu den Griechen, auf die Europa sich so gerne beruft. So heimattreu wie falsch der Topos vom "Griechischen Wunder" ist, „wir"verdanken den Griechen die Philosophie und die Demokratie in dieser Explizitheit, ebenso das Theater, so wie wir es in Europa und anderen Teilen der Welt kennen. Ob wir ihnen die Heimat verdanken, ist schwer zu sagen; gewiss geht etwas von ihr auf sie zurück. Die historische Anthropologie am Centre Louis Gernet der EHESS, allen voran Nicole Loraux (Loraux 1998), legte die Strukturen, aber auch das Unstrukturierte der griechischen Herkunfts- und Ursprungsmythen frei, insbesondere das darin kristallisierte Geschlechterimaginäre.

In diesem wird der Weiblichkeit ihr Erdcharakter, der Erde ihre Mütterlich- und Weiblichkeit nachhaltig umgehängt, die Frau wird dafür auf den vom Mann besamten Acker, die Polis auf die Patris reduziert. Mittendrin die Metropolis, wörtlich „Mutterstadt“ und Sehnsuchtsort für jene, die in der Ferne der Kolonie lebten und leben. Athen war also beides, Patris und Metropolis; Athene die Polisgöttin; Gê die Erde und Göttin der Autochthonie. Dem fügt Platon den Begriff der Chôra hinzu, was eigentlich Territorium, Land heißt, das er aber zur Mutter bzw. Amme des Werdens macht, und die er selbst als wundersamen Begriff bezeichnet, unbestimmbar weil in sich widersprüchlich, a-topos oder paratopos, das ist nicht ganz ausgemacht, jedenfalls der Logik spottend, nicht nur der platonischen (Timaios 52c-d).

Von diesem wundersamen Begriffstopos geht es zur Frage nach der geschlechtermetaphorischen und sodann nach der affektiven Seite des Paratopos Heimat, die im bereits erwähnten „Unheimlichen“ mündet.

\section{Geschlecht: Körper, Topos, Projektionsimaginäres der Weiblichkeit}

Um die Heimat in Bezug zur Geschlechtermetaphorik zu betrachten sei kurz auf eine frühere Arbeit verwiesen, die sich mit den Schichtungen des geschlechtsspezifischen Imaginären befasste, insbesondere mit dem, was ich das Projektionsimaginäre der Weiblichkeit nenne (imaginaire-écran de la féminité) (Pechriggl 1998; 1999; 2000). In modernen Staaten wird die Patria oft als weibliche Figur dargestellt, die aber nicht vorschnell als Frau interpretiert werden darf. Vielmehr greifen diese Figuren, ob Marianne für die „Nation 
France", Germania oder andere, weibliche Gottheiten auf, deren geschlechterpolitische Funktion es ist, Frauen im doppelten Sinn in jenen politischen Räumen zu repräsentieren, die Männer damit exklusiv sich selbst als herrschende Geschlechterklasse vorbehalten. Wie die heilige Barbara, die noch heute oft angerufene Schutzpatronin des Untertagbaus, duldet - so der männliche Exklusionsmythos - die verheiligte „Frau“, welche die Nation repräsentiert, keine lebenden Frauen in ihren Stollen bzw. in den Institutionen der Patris, die ausschließlich dem Vater und dessen großen Söhnen gehört, ${ }^{7}$ welche ja das patriotische Imaginäre verwirklichen (und nicht repräsentieren, wie es die allegorisch zugerichteten Pseudofrauen tun).

Ein viel älterer, politischer Frauen-Exklusionsmythos, den wir in Aischylos' Orestie finden, zeigt die Kontinuität in diesem ausschließlich männlichen Heimatimaginären an. Er besagt, dass im Kampf zwischen Poseidon und Athene um die heilige Patronanz über Athen die Göttin den Sieg davontrug, um den Preis - des Frauenwahlrechts ... Die Ver-tretung der Frauen durch eine repräsentative Göttin oder allegorische Frauenfigur wie Marianne erfüllt nun zwei Funktionen gleichzeitig: Sie hält zum einen die Frauen außerhalb der Männerräume, insbesondere in der politischen Sphäre bzw. verdrängt sie wieder aus ihr, sollten sie sich - wie etwa im Zuge der Französischen Revolution oder der Pariser Commune - in sie hineinreklamiert haben (faire écran heißt im Französischen abschirmen); zum anderen fungiert sie als Projektionsfläche (écran bedeutet auch Projektionsschirm oder Kinoleinwand) für Affekte und Vorstellungen. Die Pseudopräsentifizierung des von den patriotischen Männern angebeteten und verteidigten „Weiblichen" ist zentral für deren patriotische Euphorie, die besonders in Zeiten des Krieges erforderlich ist, wenn Männer einberufen werden, und aufgerufen sind, ihr Leben auf dem Schlachtfeld aufs Spiel zu setzen, ja zu lassen. Die Heimat als Patria ist die mütterliche Figur im Namen des Vaters, zu der die kämpfenden Söhne in ein besonders erhebendes Verhältnis treten, und durch das sie ihr Leben gleichsam als Ausgleich zum Todesrisiko der Frauen im Kindbett riskieren sollen. „Wehren und Gebären“, so das Motto der Geschlechterkomplementarität, die in diesem ideologisch-affektiv, ja nachgerade blutig aufgeladenen Heimatsetting wirkt; ein Setting, das sich gegen die Teilnahme der Frauen nicht nur durch ausagierte Frauenfeindlichkeit, sondern vor allem durch das patriotische Projektionsimaginäre der Weiblichkeit abschottet.

\footnotetext{
7 „Land der Berge, Land am Strome / Land der Äcker, Land der Dome, /Land der Hämmer, zukunftsreich! / Heimat bist Du großer Söhne, / Volk, begnadet für das Schöne, / Vielgerühmtes Österreich. / Vielgerühmtes Österreich ...." So lautete die erste Strophe der Österr. Bundeshymne, die seit 2012 inklusiver abgesungen werden muss: „... Heimat großer Töchter und Söhne“. Dagegen begehrten sofort zahlreiche männliche Volkshelden auf, die sich aus ,ästhetischen“ Gründen an der Änderung zu stoßen vorgaben. Intergeschlechtliche Menschen, die nach wie vor ausgeschlossen sind, haben sich aber meines Wissens nicht für „Heimat bist Du großer Kinder“ eingesetzt; ein solcher Vers wäre dann doch zu wahrheitsgemäß und entmystifizierend gewesen.
} 
Dabei wird die Enge und das Unheimliche der paternalistisch-familiären Geborgenheit kultiviert. Die Kleinfamilie ist der paradigmatische Topos der paratopischen Heimat. Eine Zeit lang schien es, als ob für immer in ihrer Mitte, um es mit Foucault zu sagen, die Heterotopie - diese alltäglichste aller verwirklichten Utopien - wohnen würde, ohne je zu einem weltoffenen oder gar inklusiven Gesellschaftsentwurf zu gelangen.

\section{Topologie der Affekte: Ambivalenz und Spaltung im Heimat-Imaginären}

Die Aktualisierung des Heimat-Imaginären verspricht bei jenen, die sie einmal erlebt haben, das Wieder-Aufkommen und den Erhalt kindlicher Gefühle der Geborgenheit im Schutz mütterlicher bzw. elterlicher Liebe und Fürsorge. Aber auch denen, die sie nie erlebten, wird sie gerne vorgegaukelt. Gerade in Momenten, in denen ein Kollektiv großer Angst und den damit verbundenen Ohnmachtsgefühlen ausgesetzt ist, stellt sich der Wunsch nach der heimatlichen Kombination aus Imaginärem und Stimmung als Abwehrschirm gegen die massiven Unlustaffekte ein. Mit dem in Extremsituationen durchaus üblichen Spaltungsmechanismus (Verkennung, Verleugnung, Verneinung, Verwerfung, Verkehrung ins Gegenteil, Ausblendung im Zeichen extremer Dichotomisierung) greift die menschliche Psyche auf frühkindliche Abwehrstrategien zurück, die jedoch im Fall der Chronifizierung zu Realitätsverlust führen und mit einer fabulatorischen Charakterbildung einhergehen. Eine solche Chronifizierung kann sich auch durch dauerhafte Bedrohung und ideologische Berieselung eines Kollektivs in diesem selbst einstellen.

Philosophisch-anthropologisch gesprochen ist der Mensch ein Wesen, das sich ein ,zu Hause", ein geschütztes Lager schafft/e, individuell, in Gruppen, und auch in größeren Kollektiven. ${ }^{8}$ Das können auch Zelte von Nomaden sein, wichtig ist die Schutzfunktion, der Unterschlupf, der auch noch in der Unterwerfung großdeutscher Bewohner_innen Restösterreichs unter den mächtig sich gebärdenden Nachbarn eine Rolle spielte, in dessen „Behemotschen“ Schoß Großdeutsche und später illegale Nazis rasch zu eilen suchten, und in dem sie die eigentliche Heimat sahen, frei von Anderssprachigen, frei von Juden, frei von (jüdischen) Bolschewiken, frei von Sozis.

Damit wäre der Paratopos völkischer Heimat-Rhetorik erreicht, in und durch den die erklärten Feinde im Inneren und Äußeren als „Ab-jekte“ phantasiert, angerufen und entsprechend sonder-behandelt werden. Einschluss, der den Ausschluss immer schon mit sich führt und nach sich zieht. Ein real erlebter und deshalb umso eher wieder befürch-

8 Zur frühen anthropologischen Theorie über die - präpolitische - koinônia, jene die Einzelnen schützende Gemeinschaft, zu welcher Menschen sich sammeln, siehe Aristoteles' Politik; für Ausführungen zu einem menschlichen „Trieb, sich zu betten“, der mit einer schützenden Überdachung einhergeht (Link, 1990). 
teter Verlust dieses Schutzes mobilisiert nicht nur massive Ängste, sondern auch die erwähnten psychischen Abwehren in den Einzelnen und - im Fall von Großgruppenphänomenen - im gesamten betroffenen Kollektiv. Dies führt zur Potenzierung der Affekte und entsprechenden Abwehren, was eine Spirale in Gang setzt: Die Heimat, die alle schützen soll, bedarf ihrerseits des Schutzes und guter Heimatschutz gegen bösen Nestschmutz ist das propagandistische Credo, das in Zeiten der Spaltung zur obersten Priorität gegen die Feinde im Inneren wird, welche ja noch dem realistischen Denken und Sprechen verbunden bleiben, oder um es philosophischer zu sagen: „der Wahrheitssuche“.

Und da der Mensch überhaupt, über die nationalistischen Häupter hinaus, nicht nur ein Wesen ist, das „die Sprache hat“, zoon logon echon, sondern auch ein mit Einbildungskraft begabtes, wird das Heimatphantasma stets in dieser Doppelfunktion von repräsentativer und affektiv besetzter Schutzmacht in Szene gesetzt werden: Die inneren Bilder mobilisieren die an sie gebundene affektive Entspannung und umgekehrt, die heimatlichen Gefühle evozieren die je dazugehörigen inneren Heimeligkeitsbilder, die sich ihrerseits wohlig versichernd anfühlen. Dieser geschlossene Kreislauf zwischen Geborgenheitsmise en scène und affektiver mise en acte, also das unmittelbare Ausagieren, in dem die für Selbstinfragestellung nötige Distanz tendenziell fehlt, lässt die mise en sens (Sinnstiftung durch kritisches Nachdenken) gar nicht oder nur schwer aufkommen. Die affektive mise en acte im Register des Heimatimaginären ist vielmehr das unvermittelte kollektive Ausagieren des Fremdenhasses, bei dem der Fremde immer schon fein säuberlich in den anderen verlegt zu sein scheint. Das hervorzurufen, dazu tendiert die Heimat stets; darin besteht ihre imaginäre wie affektive Verstetigung, so wie die Religion aber auch die herrschaftliche Staatsideologie zum total(itär)en Fanatismus tendiert, weil sie als Glaube an das Ganze alles verneinen muss, was sich ihm nicht fügt. Und im Zeichen der Spaltung stehend dämmert in der Heimat, noch bevor sie als patris/Vaterland zum imaginären und dann realen Kriegsschauplatz aufsteigt, schon das genaue Gegenteil der Heimeligkeit, die sie verspricht: zutiefst Unheimliches. Denn bevor die Spaltung aktiv wird, liegen die Gegensätze miteinander verschränkt am Grund des heilversprechenden Heimatscheins, so wie „die Ambivalenz der Urworte“ am Grund des Unbewussten liegt.

Es soll dort zwar so schön und einhellig sein, wie es - außer in der nachträglich idyllisierenden Erinnerung - nie war, doch das ist nur die eine Seite. Dieses Imaginäre erstrahlt für Bloch als Vorschein bzw. im Nachschein idealer Vergangenheiten, die bei Marx „urkommunistische“ Retroutopien sind. Und so ist die ganze Menschheit in diesen Idyllen zumindest in der Vorstellung heimelig eingebettet zwischen Damals und Morgen. Tendenziell verklärende Heilslehren einer politisch freien und gleichen Heimat im Anschluss auch an antike Gemeinden, die Französische Revolution oder die Pariser Com- 
mune finden sich nicht nur bei Marx, Bloch und Benjamin, sondern auch bei manchen Anarchist_innen und Feministinnen; wir finden sie nicht bei Luxemburg, die zwar auch in der genannten Tradition nach der besten aller Regierungsformen strebte, jedoch „,nach Möglichkeit" und effektiv radikaldemokratisch, weniger in dem messianischen Glauben an etwaige Meisterrepräsentanten, also daran, dass das Paradies auf Erden sich als universelle, ja nachgerade totale Heimat (Blochs „Totum Bonum“) erfüllen würde. Die Erfüllung soll sich da von selbst bzw. dem Gesetz der Geschichte folgend, oder abgeleitet vom „Prinzip Hoffnung" durch die versöhnt-versöhnende Theorie-Praxis einstellen, wenn sie etwa von Bloch mit dem erfüllten Augenblick herbeigeträumt wird, und zwar als heiliger Kairos, nicht als jener realistische und praktische kairos aus Medizin (Hippokrates) und Politik (Aristoteles), den es als nur kurz währende Gelegenheit zu ergreifen gilt.

Die idealisierenden Vorstellungswelten gepaart mit einer psychologisch-anthropologischen Perspektive legen nahe, dass Heimat etwas Universelles sei, zumal wir auch von „politischer Heimat“ sprechen, die sich im Kosmopolitismus Zum ewigen Frieden (übrigens auch Name eines Wirtshauses) auf alle Menschen ausbreiten würde, wenn es nach Kants - durchaus auch ironischer - Geschichtsphilosophie ginge. Die „Neger“ (Kant) waren davon gewiss ausgenommen; hier blieb Kants Weltoffenheit, sein bis heute viel gepriesener Humanismus, regional eingeengt, sprich eurozentrisch-rassistisch und gewiss auch kleinbürgerlich, was sich mit seiner süffisanten Frauenfeindlichkeit gut verbinden ließ. ${ }^{9}$

Wenn nun zwar die Ideen universeller Bürgerschaft paratopisch, bei/nahe und wider die je kulturellen Abschottungstendenzen, alle humanistisch-heimatlich einzuschließen vermögen, so gilt auch hier: Der Ausschluss ist konstitutiv für den Einschluss, und auf die Manifestation der fein säuberlich Ausgeschlossenen folgt ihre Abspaltung, d.h. die Abspaltung der „Anderen“ vom „Eigenen“. Damit verbunden ist aber auch die umgekehrte Peinlichkeit, dass mit dem Wegfall dieses konstitutiven und stets für Verunglimpfungen bereiten „Anderen“ aus dem bewussten Wahrnehmungsraum ein fundamentales Loch im „Eigenen“ entsteht, das sogleich wieder gefüllt werden muss: Ein anderer Anderer muss her, und sei er durch Identifikation illusionär herbeigeschafft. Das geschieht auch durch etwas, das ich als Self-Othering bezeichnen möchte: „Wir sind die eigentlichen Opfer" sagen z.B. die ihres konstitutiven Anderen sich beraubt habenden Täter; oder die Täter_innen, die auf der Betrachtungsebene der Einzelpsyche vielleicht tatsächlich chronisch Opfer waren, identifizieren sich mit den Opfern, indem sie in Ermangelung jeglichen Sinns für Verhältnismäßigkeit etwa die Pflicht, in Zeiten der Pandemie Mas-

9 Das heißt keineswegs, dass wir aufhören könnten, ihn zu lesen, oder seinen Humanismus ernst zu nehmen, wie manche es neuerdings minoritätsidentitär - und keineswegs mehr „dissidentitär“ - fordern. Ich kann aus Platzgründen darauf nicht genauer eingehen. (Pechriggl 2016). 
ken zu tragen mit der Vergasung in einem NS-Vernichtungslager gleichsetzen, was auf eine besonders pikante Kombination von Spaltungsmechanismen verweist.

Diese korreliert mit identitärer Politik im höchst zugespitzten Sinn von Freuds „Narzissmus der kleinen Unterschiede", wenn nicht gar im Sinn kollektiver Paranoia, der auch wieder nur mit humanistisch-internationalistischen Orientierungen zu entkommen ist, welche auf der Erfahrung und dem Begriff der Interdependenz basieren. Alles andere als hilfreich ist dagegen der abstrakte Begriff, gegen dessen Dominanz die Partikularismen ja gerade aufbegehren. Es geht nur durch die möglichst gleichberechtigte Konkretisierung des Allgemeinen, das wusste schon Hegel auszudrücken und das versucht auch das Ringen um gesellschaftliche Bezeichnungen von Diskriminierten wie LGBTIQ*, wobei das * für den nie erfassbaren Rest, die nächsten, noch nicht zu Klassen- bzw. Gruppenbewusstsein gelangten Diskriminierten, Unterdrückten, Ausgebeuteten steht. Dass dagegen der spaltende kollektivpsychische Topos (diesmal im Sinn von Allgemeinplatz) des Self-Othering gerade im Heimat-Imaginären der Dominanzkultur virulent wird, wissen wir aus diversen sozialpsychologischen Analysen der Kriege und ethnischen Konflikte seit Freud (Freud 1927). Er kann aber auch Minderheiten erfassen.

\section{Zeit/Raum: transtopische Überschreitungen}

Wenn wir allerdings die von immer wiederkehrender Ent-täuschung begleiteten Heimatillusionen auf ihre Verbindungen zum Herkunftsmythos hin betrachten, der den imaginierten Beginn von Gesellschaften oder auch Staaten erzählt, dann fällt eines auf: Die Spaltung als psychische Abwehr prägt nicht nur dieses Anfangskapitel der Parazeit Heimat (so wie sie die Form jeder heftigen Auseinandersetzung prägt), sie strukturiert es vielmehr im starken Sinn. Um genauer zu sein, strukturiert sie den Übergang vom ersten Gründungsereignis zum zweiten Moment der ersten Differenzierungen. Man kann derartige Phänomene auch in psychoanalytisch arbeitenden Großgruppen beobachten, wo vor allem zu Beginn die Angst (in) der Gruppe die konstitutiven Anfangsillusionen und -erwartungen massiv bedroht und dadurch zu paranoid wirkenden, fragmentarischspaltenden Wortmeldungen (auch Präsidentengezwitscher) führt. In der Realität staatlich verfasster und militärisch veranlagter Heimaten kann es da schon zum Ausagieren bürgerkriegsartiger Aufspaltung der Bevölkerungen oder kriegerischer Handlungen gegenüber einem Außenfeind kommen, der immer auch den Vorteil hat, eine Zeit lang für innere/n Frieden und Sicherheit zu sorgen, also der sich als Widerstreit zu manifestieren beginnenden Ambivalenz im „Inneren“ Herr zu werden. Das trifft auch auf die gerade noch geeinte Heimatstimmung zu: droht sie im Angesicht größerer Bedrohung einmal ins Alptraumhafte zu kippen, wird geleugnet, verteufelt, bezichtigt. Denen, die in diesem 
Modus vorstellen und denken, erscheint nur noch der für Außenstehende stets kasperlhafte Held in der Lage, das Ungeheuer zur Strecke zu bringen (oder „,wegzukickeln“), das die identitär-utopisch aufgeblähte Heimat bedroht, das er aber immer auch selbst ist, sprich diejenigen, die sich mit ihm identifizieren und durch ihn voll und ganz - total repräsentieren lassen: „einer für alle - alle für einen“.

Weil sie imaginär ist, also zuerst in der Vorstellungswelt existiert, muss Heimat sich in der res extensa materialisieren, realisieren: im Körper, im Raum, im Territorium; aber manche finden ihre Heimat eher in der Sprache, in der Dichtung, der Wissenschaft, der Philosophie; sie wirken zuweilen auf die heimatlich Verankerten, die der ländlichen, familiär-paternalistischen oder nationalen Heimat effektiver verbunden sind, wie Verlorene, bedrohliche Zugvögel inmitten einer Pseudostabilität, Migrant_innen des Geistes oder im Geiste, nomadisch nennen manche es in Anlehnung an die Lebensweisen der Beduinen, Tuaregs, Mongolen u.v.a., die vielleicht auch schon auf Elemente des Kosmopolitismus verweisen.

Wenn wir den Eingangs skizzierten Paratopoi einiger Philosoph_innen die Kosmopolitismus-Mimikri der arisch-deutschen Intelligentsia im NS-Reich gegenüberstellen, dann finden wir, ohne lange suchen zu müssen, das einzige Manifest der „deutschen Professorenschaft“, das zudem in fünf Sprachen an die „Gebildeten der Welt" gerichtet war: das „Bekenntnis der Professoren an den deutschen Universitäten und Hochschulen zu Adolf Hitler und dem Nationalsozialistischen Staat“ (NS-Lehrerbund, 1933).300 - auch einige Dozent_innen und Lehrbeauftragte haben unter diesem Titel unterzeichnet - reihen sich ein hinter die sieben Ansprachen für diesen deutschen Ruf des Professor-Seins, unter deren Autoren der Heimat- und Schollespezialist Heidegger ebenso prominent wie geistlos hervorsticht. ${ }^{10}$

Die durch Heideggers Nazi-Verstrickungen in Verruf geratene Phänomenologie und Existenzphilosophie hat interessanterweise noch während des Zweiten Weltkriegs in Frankreich zu überleben begonnen. Und es mag der Ironie des Ideenschicksals geschuldet sein, dass gerade Emmanuel Lévinas, der gegen Lebensende immer mehr zum Thora-Interpreten wurde, hier eine initiale Rolle spielte. Vor allem der Philosoph der Weiblichkeit als Andersheit und „Bleibe“ (auch eine Heimat?), Jean-Paul Sartre und in gewisser Weise auch Maurice Merleau-Ponty waren es, die Heidegger in Frankreich, Europa und darüber hinaus wieder salonfähig machten. ${ }^{11}$ Heidegger als ambivalent besetzter Star der franzö-

\footnotetext{
${ }^{10}$ Heideggers Kollege Gadamer war „nur“ unter den Unterzeichnern.

${ }^{11}$ Wie Emmanuel de Saint Aubert aufzeigt, wird diese Ambivalenz deutlich bei einer systematischen Analyse von Merleau-Pontys Bezugnahmen auf Heidegger (de Saint Aubert, 2011).
} 
sischen Postmoderne, das hört bis heute nicht auf, mich zu verwundern. ${ }^{12}$ Wenn man den fundamentalnationalistischen Wahlwerbetext von Heidegger für Hitler liest, ${ }^{13}$ besticht die Nähe zur politischen Debilität, die mit Teilen dieser Strömung in den 1980er Jahren einher ging und deren Folge der Aufstieg zunehmender identitärer Faschisierung war.

Vielleicht wird jemand dieses Kapitel französischer und europäischer Geistesgeschichte eines Tages im Lichte des französischen (de Gaules') Antiamerikanismus lesen und uns besser verständlich machen. Es wird jedenfalls nicht abgelöst zu sehen sein von den Entwicklungen der Geopolitik und anderer europäischer „Heimaten“ der Philosophie nach dem Zweiten Weltkrieg.

Doch obwohl es der politischen, gesellschaftlichen und kulturellen Voraussetzungen für Entstehung und Weiterentwicklung der Philosophie bedarf, ist die Heimat der Philosophie als Begriffswelt die Sprache. Das hat sie mit der Dichtung gemein, die sie aber aufgrund ihres Hangs zur Episteme auf Distanz hält. Dass die sprachliche Heimat der Philosophie keine lingua franca sein kann, hat schon Cicero gewusst, der die griechischen Begriffe ins Lateinische übersetzte; und auch Kant, der so viele Begriffe aus dem Lateinischen ins Deutsche übertrug, um erstmals systematisch Philosophie in dieser, seiner Muttersprache schreiben zu können. Was nun aber - philosophisch gesprochen - „Heimat" ist, ob Mythos, Begriff oder Phänomen, und inwiefern alle drei, das kann weiterhin nur an der Schnittstelle von philosophischer und historiographischer, kulturtheoretischer und sozialpsychologischer Betrachtung erhellt werden. Caspar cas und step by step, nicht ohne den ständigen Versuch, wieder alles Mögliche - von Neuem und provisorisch - auf und unter die allgemeineren Begriffe zu bringen, also ohne den Begriff erneut zum

\footnotetext{
${ }^{12}$ So wie sich die Grande Nation über die Verbrechen ihrer Armee in den Kolonien, vor allem während des Algerienkrieges hinwegtäuschte (Vidal-Naquet 2001), so machten sich seine Vorzeigeintellektuellen lange Zeit Illusionen über die Unverfänglichkeit der Heideggerschen Sprache und die Gewalttätigkeit seines politischen Denkens und Tuns. Während Derrida mit den Mitteln der Dekonstruktion auf Französisch kaum imstande ist, dem angemessen beizukommen, schlägt Elfriede Jelineks permanente Heidegger-Parodie gewaltig gegen den Blut- und Bodendünkel des Heimatphilosophen ein; ihre grausame Parodie durch Verarbeitung nicht nur seiner eigentümlichen Philosopheme, sondern auch des privaten und zugleich völkischen Duktus', ist für sprachmusikalische Ohren bei/nahe eine Tortur, bevor sie zum Genuss an der Parodie selbst wird. Vielleicht ist das ja die Hoffnung mancher deutschsprachiger Heidegger-Rezipient_innen: dass das metaphysische Getöns durch beständige Gestaltung zum philosophischen Kunstwerk werde. Ich fürchte, dass das Gelingen eines solchen Unterfangens der Literatur oder dem Theater vorbehalten ist bzw. einer Mischung dieser beiden mit der philosophischen Untersuchung, wie Platon sie so unnachahmlich vorlegte.

13 „Das deutsche Volk ist vom Führer zur Wahl gerufen. Der Führer aber erbittet nichts vom Volke, er gibt vielmehr dem Volke die unmittelbarste Möglichkeit der höchsten freien Entscheidung, ob das ganze Volk sein eigenes Dasein will, oder ob es dieses nicht will. Das Volk wählt morgen nichts Geringeres als seine Zukunft." (Heidegger 1933, 13) Ganz oder gar nicht, Existenz oder Vernichtung, Führerprinzip oder Untergang ... Heidegger ist schon ganz eingestimmt auf die pathetische Denkzwang- und KurzschlussRhetorik Hitlers.
} 
Verbrecher am Besonderen werden zu lassen. Und die Systematik der philosophischen Sprachheimat, die das System hinter sich lässt, wäre ein Begriffsgefüge, das zugleich ein Begriffsmobile ist, dessen sich alle, die möchten, bedienen sollen - ohne Patente, ohne property, öffentlich zugänglich eben, wie diese Zeitschrift mit dem schönen Namen.

\section{Literatur}

Benjamin, Walter. 1982. Das Passagenwerk. Gesammelte Schriften Band 5.1 und 5.2. Frankfurt am Main: Suhrkamp.

Bloch, Ernst. 1984. Das Prinzip Hoffnung. 3 Bände. GW Band 5. Frankfurt am Main: Suhrkamp. Castoriadis, Cornelius. 1981. Durchs Labyrinth. Hamburg: Europäische Verlagsanstalt.

Castoriadis, Cornelius. 1984 (1975). Gesellschaft als imaginäre Institution. Frankfurt am Main: Suhrkamp.

De Saint Aubin, Emmanuel. 2011. „Merleau-Ponty face à Husserl et Heidegger: illusions et rééquilibrages." Revue germanique internationale: illusions et rééquilibrages 13. Aufgerufen am 15. April 2021. http://journals.openedition.org/rgi/1122; DOI: https://doi.org/10.4000/rgi.1122.

Foucault, Michel. 2001 (1984). Schriften. Band 1. 1954-1969. Frankfurt am Main: Suhrkamp.

Freud, Sigmund. 1990 (1927). Zukunft einer Illusion. GW Band XIV, Frankfurt am Main: Fischer, 325-380.

Link, Jürgen. 1990. „Massendynamik und As-Sociation.“ kultuRRevolution 36: 3-12.

Loraux, Nicole. 1998. „Falsche Mimesis - richtige Mütter. Warum die Mütter angeblich die Erde nachahmen“ In Mutterwitz. Das Phänomen Mutter - Eine Gestaltung zwischen Obnmacht und Allmacht, herausgegeben von Gudrun Perko, 29-51. Wien: Milena.

NS-Lehrerbund (Hg.). 1933. Bekenntnis der Professoren an den deutschen Universitäten und Hochschulen zu Adolf Hitler und dem Nationalsozialistischen Staat. Dresden. Abgerufen am 10. April 2021. https://archive.org/details/bekenntnisderpro00natiuoft/page/n3/mode/2up

Pechriggl, Alice. 1998. „Welche Mutter? Überlegungen zur Geschichtung einer ambivalenten Gestalt zwischen Psyche-Soma und Gesellschaft." In Mutterwitz. Das Phänomen Mutter - Eine Gestaltung zwischen Ohnmacht und Allmacht, herausgegeben von Gudrun Perko, 339-377. Wien: Milena.

Pechriggl, Alice. 1999. „Der Körper in den Gestaltungen und Schichtungen des geschlechtsspezifischen Imaginären." In Körper-Konzepte, herausgegeben von Julika Funk und Cornelia Brück, 25-35, Tübingen: Gunter Narr.

Pechriggl, Alice. 2000. Corps transfigurés. Stratifications de l'imaginaire des sexes/genres. 2 Bände. Paris: l'Harmattan.

Pechriggl, Alice. 2016. „Sprachregelung und Verfügung über die Vergangenheit." DERDIEDAS. Akademiezeitung 5: 5-6.

Safran, William. 1991. „Diasporas in Modern Societies: Myths of Homeland and Return.” Diaspora: A Journal of Transnational Studies 1 (1): 83-99.

Vidal-Naquet. Pierre. 2001 (1975). Les crimes de l'armée française. Algérie 1954-1962. Paris: La découverte. 